\title{
B2.5-Eunomia simulations of Pilot-PSI and Magnum-PSI plasmas
}

\author{
R. C. Wieggers ${ }^{\mathrm{a}, *}$, D. P. Coster ${ }^{\mathrm{b}}$, P. W. C. Groen ${ }^{\mathrm{a}}$, H. J. de Blank ${ }^{\mathrm{a}}$, W. J. Goedheer ${ }^{\mathrm{a}}$ \\ ${ }^{a}$ FOM Institute DIFFER - Dutch Institute for Fundamental Energy Research, Association EURATOM-FOM, Partner in the Trilateral \\ Euregio Cluster, the Netherlands, www.differ.nl \\ ${ }^{b}$ Max-Planck-Institut für Plasmaphysik, EURATOM Association, Garching, Germany
}

\begin{abstract}
The B2.5-Eunomia code is used to simulate the plasma and neutral species in and around a Pilot-PSI plasma beam. B2.5, part of the SOLPS5.0 code package, is a multi-fluid plasma code for the scrape-off layer. Eunomia is a newly developed non-linear Monte Carlo transport code that solves the neutral equilibrium, given a background plasma. Eunomia is developed to simulate the relevant neutral species in Pilot-PSI and Magnum-PSI, linear devices that study plasma surface interactions in conditions expected in the ITER divertor. Results show the influence of the neutral species on the Pilot-PSI plasma beam. We show that a fluid description for the neutrals is not sufficient and Eunomia is needed to describe Pilot-PSI. The treatment of individual vibrational states of molecular hydrogen as separate species is crucial to match the experiment.
\end{abstract}

Keywords: Divertor modelling, Molecular effects, Neutral modelling, Edge modelling

\section{Introduction}

A newly developed parallel non-linear three dimensional Monte Carlo code, called Eunomia[1] has been coupled to B2.5[2] to investigate the role of neutral species in the linear plasma generator Pilot-PSI[3]. The plasma conditions in Pilot-PSI and its successor Magnum-PSI[4], with plasma densities of $\approx 5 \times 10^{20} \mathrm{~m}^{-3}$ and plasma temperatures below $5 \mathrm{eV}$, are similar to those expected in the ITER divertor. The plasma described here differs significantly from that in other linear machines like Pisces[5], PSI2[6], and NAGDIS[7]. Those experiments typically have plasma densities of around $10^{19} \mathrm{~m}^{-3}$ and a plasma temperature around $10 \mathrm{eV}$. The design of the source and the plasma conditions in Pilot-PSI and Magnum-PSI result in a much higher production of neutrals. This implies that Pilot-PSI enters a different, interesting regime, where the mean free path of neutrals is short enough to strongly influence the plasma. On the other hand, the neutral mean free path is long enough that neutrals will face changing plasma conditions in between their collisions. This requires a kinetic description for the neutral species.

Previous simulations with the coupled B2-Eirene code[2] addressed plasma conditions expected in Magnum-PSI, assuming higher temperatures than observed in Pilot-PSI[8]. The simulations performed with B2.5-Eunomia are in a high density, low temperature regime that is more challenging, both in the complexity of the physics and the statistical demands.

Eunomia is developed to model the behaviour of neutral species under these high density, low temperature con-

${ }^{*}$ Corresponding author E-mail: R.C.Wieggers@differ.nl ditions in detail. The code can be applied to both linear machines and tokamaks. Eunomia calculates the particle density, temperature and flow of the relevant neutral species by simulating collisions with ions, electrons, neutrals and the surrounding walls. The physics included in Eunomia is similar to other neutral transport codes like Eirene and Degas 2[9;10]. In Eunomia special attention is paid to improving the work load balance for parallelisation and to reduce statistical noise, in particular near the symmetry axis, by applying cell based adaptive particle weights.

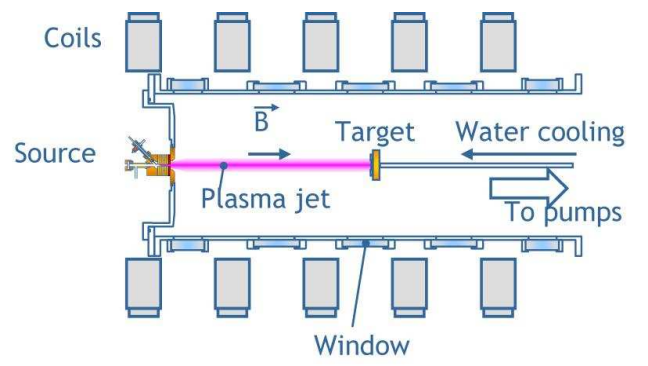

Figure 1: Schematic drawing of Pilot-PSI, with the cascaded arc source on the left and target on the right.

In this paper we report on the importance and behaviour of atomic and molecular hydrogen in and around a Pilot-PSI plasma beam. The B2.5-Eunomia simulations are compared to experimental results. Figure 1 shows a schematic drawing of Pilot-PSI. The inlet plasma boundary conditions are taken from Thomson scattering measurements near the source. Simulation results are compared to Thomson scattering profiles near the floating tar- 
get. We also applied a negative bias to the target to draw an ion current. The potential at which the ion saturation current is reached is compared to measurements in PilotPSI.

\section{B2.5}

B2.5, part of the SOLPS5.0 code package, is a multifluid two temperature plasma code for simulation of the scrape-off layer and divertor of a tokamak. B2.5 solves five equations: The electron and ion heat equations, the continuity, parallel momentum and potential equations. The drift velocities and electric currents are included. For the cylindrically symmetric geometry of Pilot-PSI extensions are made to the inlet and target boundary conditions. At the inlet we implemented an option to apply radial profiles for the ion and electron temperatures, the potential and the density or particle flux density as boundary condition. At the target we implemented a floating target boundary condition. To obtain a given total current, the target potential is adjusted during the simulation.

\section{Eunomia}

Eunomia solves the equilibrium state of each neutral species. The plasma and neutral background is specified as cell averaged values on a tetrahedral grid. Eunomia calculates a new neutral background characterised by the density, flow velocity and temperature of each neutral species. Eunomia converges by iteratively updating the neutral background.

Eunomia simulates collision between test-particles and particles drawn from the local background. The rates of neutral-electron collisions are a function of the local electron density and temperature. The neutral-neutral and neutral-ion collisions are simulated in the center of mass frame. For those heavy particle collisions we use cross sections and apply the null collision method. Rates and cross sections for collisions with charged particles and volume recombination are taken from the same databases as used by Eirene and BIT $[9 ; 11 ; 12]$. For collisions between neutral species the BGK approximation is used [13].

Atomic and molecular hydrogen have elastic collisions with atomic and molecular hydrogen, and with protons. For atomic hydrogen we also consider charge exchange $\left(\mathrm{H}^{+}+\mathrm{H} \rightarrow \mathrm{H}+\mathrm{H}^{+}\right)$, excitation $\left(\mathrm{e}+\mathrm{H} \rightarrow \mathrm{e}+\mathrm{H}_{\mathrm{n}=2}^{*}\right)$ and ionization $\left(\mathrm{e}+\mathrm{H} \rightarrow 2 \mathrm{e}+\mathrm{H}^{+}\right)$. Excited hydrogen atoms are not simulated in Eunomia. Based on a collisional radiative model we determine the ionization probability and ionize or deexcite the atom instantaneously.

Charge exchange with molecular hydrogen produces a molecular ion, which is assumed to recombine instantanously $\left(\mathrm{H}^{+}+\mathrm{H}_{2} \rightarrow \mathrm{H}+\mathrm{H}_{2}^{+}\right.$; e $\left.+\mathrm{H}_{2}^{+} \rightarrow \mathrm{H}_{\mathrm{n}>2}^{*}+\mathrm{H}\right)$. At the low plasma temperature of Pilot-PSI this process, called molecule activated recombination (MAR), recombines the plasma efficiently. Dissociative attachment produces a negative ion, which is assumed to recombine instantaneously $\left(\mathrm{e}+\mathrm{H}_{2} \rightarrow \mathrm{H}+\mathrm{H}^{-} ; \mathrm{H}^{-}+\mathrm{H}^{+} \rightarrow \mathrm{H}+\mathrm{H}_{\mathrm{n}=3}^{*}\right)$. In case vibrational states are treated as separate species, electron impact vibrational excitation and deexcitation are also considered.

\section{Coupling of B2.5 and Eunomia}

The coupling of B2.5 and Eunomia is done by linking the codes into one executable. B2.5 can access Eunomia data internally. The modifications to both codes are very limited and in such a way that the codes can still be used independently.

Based on the plasma background from B2.5, Eunomia calculates sources of plasma particles, ion parallel momentum and ion and electron heat. Collisions between neutrals and the plasma contribute to these sources. For instance, an ionization event is a source of one ion and electron, and an electron energy sink equal to the ionization potential. The velocity vector of the neutral particle will be used to determine the ion heat and ion momentum source. The sources in a certain cell are given by

$$
\begin{aligned}
& S_{P}=\sum_{i} \Delta P_{i}, \\
& S_{M}=\sum_{i} m\left(\mathbf{v}_{1}-\mathbf{v}_{0}\right), \\
& S_{H}=\sum_{i} \Delta \frac{1}{2} m\left(\mathbf{v}_{1}-\mathbf{u}\right)^{2}-\frac{1}{2} m\left(\mathbf{v}_{0}-\mathbf{u}\right)^{2}, \\
& S_{E}=\sum_{i} \Delta E_{i},
\end{aligned}
$$

where $i$ is the collision event. Here $\mathbf{u}$ is the ion background velocity and $\mathbf{v}_{0}$ and $\mathbf{v}_{1}$ are the velocity vectors of the ion before and after the collision event. $\Delta P_{i}=-1,0$ or 1 and $\Delta E_{i}$ equals the electron energy gain or loss for the collision event. For example: $\Delta P_{i}$ equals minus one for charge exchange of a proton and a hydrogen molecule, zero for charge exchange with a hydrogen atom and one for ionization.

B2.5 also provides Eunomia with ion fluxes to surfaces. Surface recombination is simulated by a neutral source in Eunomia. B2.5, like most fluid codes, does not resolve the sheath, but simulates up to the sheath entrance. Eunomia requires the ion temperature $T_{i}$, the sheath potential $V_{s h}$ and the parallel ion velocity $v_{\|}$from B2.5 to determine the average energy of neutral particles leaving the target:

$$
E_{n}=\alpha\left(\frac{3}{2} T_{i}+V_{s h}+\frac{m_{i}}{2 e} v_{\|}^{2}\right),
$$

where $\alpha$ is the energy reflection coefficient.

Typically every 10 time-steps B2.5 calls Eunomia. On the updated plasma background Eunomia calculates a new neutral background and updates the source terms for the B2.5 equations. Recursively this will converge to the B2.5Eunomia steady state solution. 


\section{Results and discussion}

We will compare B2.5-Eunomia simulation results to Pilot-PSI experiments at a magnetic field of $0.8 \mathrm{~T}$. Under the same conditions Thomson scattering measurements near the source and near the target were made. The lateral electron density and electron temperature profiles obtained from the Thomson scattering measurements near the source are used as inlet boundary conditions for B2.5 (side 1 in Figure 2). At this boundary a potential profile derived from rotation measurements is applied. At the target (side 2 in Figure 2) B2.5 sheath conditions are applied.

One of the sources in Eunomia is volume recombination by two and three body recombination. Another neutral source is the surface recombination of the ion flux to the target. The cascaded arc source gas flow was $1.5 \mathrm{slm}$ and reaches an efficiency of about $10-15 \%$. The neutral particles released by the source are accounted for by a gas puff near the inlet of the computational domain (side 4 in Figure 2). At side 5 in Figure 2 a small fraction of test-particles is absorbed, simulating pumping. The absorption probability is automatically adjusted to obtain the measured background gas pressure of $2.4 \mathrm{~Pa}$.

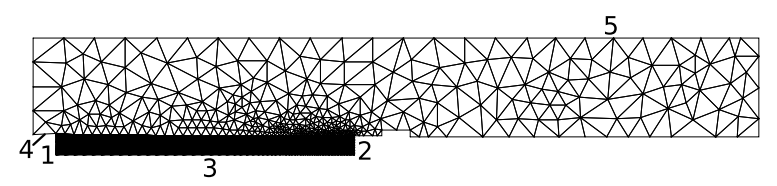

Figure 2: The two dimensional projection of the grid in the $(r, z)$ plane. 1: inlet at position of Thomson scattering. 2: target. 3: axis. 4: position of neutral source from the cascaded arc. 5: pump.

When impinging on walls, atoms reflect as atoms or associate to molecules. Due to dissociation and MAR two or three atoms per molecule are produced. The balance of the sources and sinks due to pumping, volume recombination, wall interactions and collisions lead to an equilibrium density, temperature and flow profile for each neutral species.

Figures 3 and 4 compare the radial profiles of the electron density and temperature obtained from B2.5-Eunomia with the Thomson scattering measurements near the target. For the B2.5 standalone case atomic hydrogen is simulated as a fluid. This model cannot describe all the important physics in Pilot-PSI conditions correctly. We were not able to apply it to a reasonably high neutral pressure of a few Pascal. The lower neutral background pressure explains the high electron temperature and density for this case.

In the case of B2.5-Eunomia with atomic hydrogen only, the simulated temperature fits the Thomson measurements very well. The plasma is effectively cooled by the efficient and frequent charge exchange collisions with atomic hydrogen. However, the peak density is a bit too high, and the density profile is much broader than in the experiment. There is no atomic hydrogen process that recombines the plasma. This leads to a higher density than

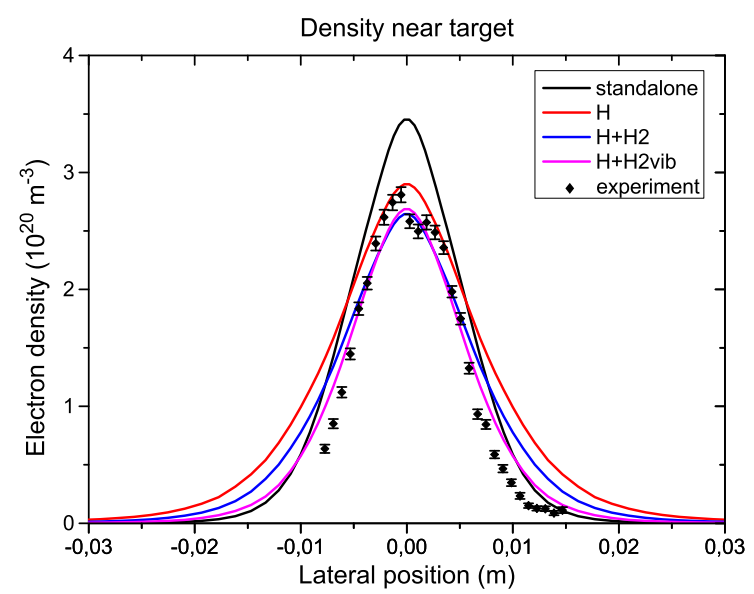

Figure 3: Thomson scattering measurements of the electron density near the target compared with results of B2.5 standalone and 3 cases of B2.5-Eunomia are shown.

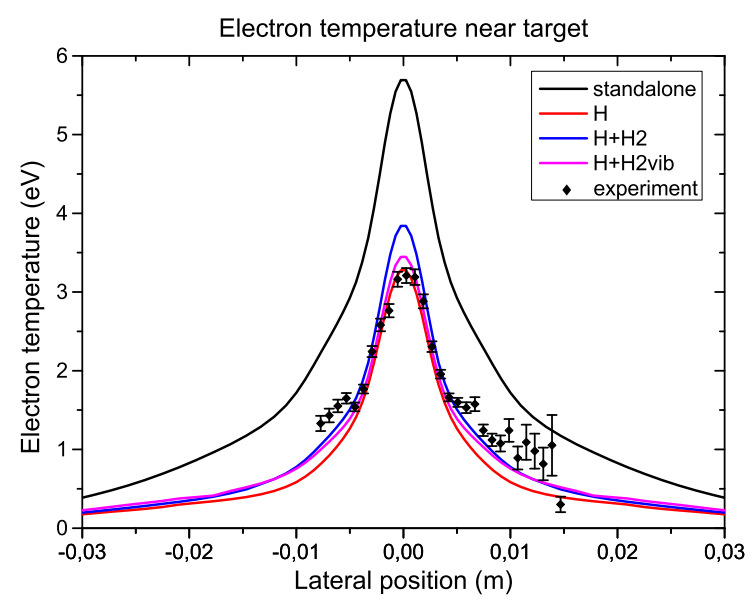

Figure 4: Thomson scattering measurements of the electron temperature near the target compared with results of B2.5 standalone and 3 cases of B2.5-Eunomia are shown.

in the experiment.

When molecular hydrogen is included as single species in the Eunomia calculations, while keeping the neutral pressure at the same value, the density of atomic hydrogen will drop. This will cool the plasma less, as shown by the blue line in Figure 4. Figure 3 shows that molecular hydrogen, formed by association of atomic hydrogen at the vessel wall, will decrease the plasma density via the MAR process.

The best fit with the experiment is obtained when simulating all vibrational states of the hydrogen molecule as separate species. The peak values of the two radial profiles match within the uncertainties of both the experiment and the code. At the edge of the temperature profile, the Thomson scattering profile is higher. However, the uncertainty of the Thomson scattering measurements at the edge of the profile is large due to the lower plasma density, thus lower scattering signal. Especially for the density, the width of the plasma profile is best reproduced by B2.5- 


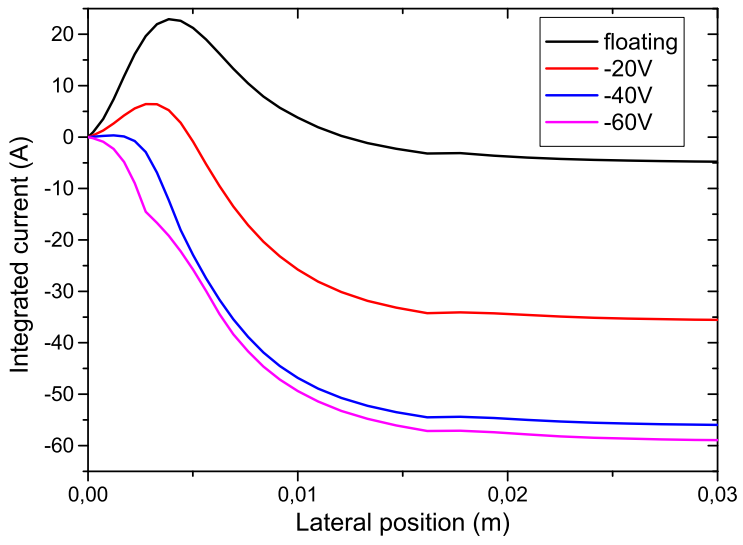

Figure 5: Integrated current for different target potentials.

Eunomia simulations including vibrational states of molecular hydrogen as separate species. Charge exchange with molecular hydrogen is very efficient around the vibrational states 3 and 4 . The distribution over the vibrational states of molecular hydrogen produced at the wall is according to [14]. Combined with the vibrational excitation by electron impact collisions, the vibrational levels 3 and 4 of hydrogen molecules at the edge of the beam are well populated. Therefore, more plasma at the edge of the beam will recombine, resulting in a reduced width of the plasma beam. The simulated density profile is still broader than the Thomson scattering profile. This indicates that recombination via vibrational excited hydrogen molecules is still underestimated in these simulations. Higher vibrational states are lost in charge exchange and dissociative attachment. Therefore, the net effect of the electron excitation and deexcitation cools the electrons. A second explanation for the temperature drop compared to the simulations of molecular hydrogen as a single species is that the plasma beam acts as a sink for vibrationally excited molecules and source of atomic hydrogen. This source of atoms and sink of molecules when simulating the vibrational states as separate species leads to a higher atomic hydrogen density, thus more efficient cooling by atomic hydrogen.

Figure 5 shows the integrated current as function of radius when biasing the target negatively. The nonzero gradient at $r<0.015 \mathrm{~m}$ shows that there are currents in the plasma beam. In the simulations we found a floating potential of around $-8 \mathrm{~V}$. When biasing the target negatively, at some potential one should reach the ion saturation current. Figure 5 shows that at a target potential of $-40 \mathrm{~V}$ the ion saturation current has almost been reached. In the experiment, the floating potential was around $-27 \mathrm{~V}$ and we reached the saturation current at $-60 \mathrm{~V}$. There is a clear mismatch between the simulations and the experiments. However, the difference between the floating potential and target potential at saturation is in both the experiment and B2.5-Eunomia simulations just over $30 \mathrm{~V}$.

In the experiment, the saturation current was around $-30 \mathrm{~A}$. The total current through the target at the ion sat- uration current as calculated by B2.5-Eunomia is a factor 2 too high. This can partially be explained by the broader beam in the simulations. The influence of the prescribed potential profile at the inlet can also be an effect. This profile is based on rotation measurements of neutrals. The ExB rotation, and therefore also the electric field and potential, might be underestimated, since the neutrals do not rotate as fast as the ions. The influence of the beam width and the inlet potential profile on the target potential and current through the target has to be studied in more detail.

\section{Conclusions}

The coupled B2.5-Eunomia has successfully been applied to the linear geometry of Pilot-PSI. The experimentally obtained density and temperature profiles can be reproduced, when vibrationally excited hydrogen molecules are considered as separate species in Eunomia. The vibrationally excited hydrogen molecules cool the plasma and efficiently recombine the plasma at the edge of the beam. The narrow Thomson scattering profile of the density indicates that the effect of vibrationally excited hydrogen molecules is still underestimated. The potential difference between floating potential and ion saturation current matches the experiments perfectly. However, the simulated floating potential differs $20 \mathrm{~V}$ from the experimental value. The sensitivity of the potential boundary condition and the beam width have to be studied in detail to solve this.

\section{Acknowledgement}

This work, supported by the European Communities under the Contract of Association between EURATOM/FOM, was carried out within the framework of the European Fusion Programme with financial support from NWO. The views and opinions expressed herein do not necessarily reflect those of the European Commission.

\section{References}

[1] R. C. Wieggers and et. al., Contributions to Plasma Physics (accepted in 2011).

[2] R. Schneider and et. al., Contributions to Plasma Physics 46(1-2), 3-191 (2006).

[3] G. J. van Rooij and et. al., Applied Physics Letters 90(12), 121501 (2007).

[4] J. Rapp and et. al., Fusion Engineering and Design 85(7-9), 1455-1459 (2010).

[5] R. P. Doerner, D. G. Whyte, and D. M. Goebel, Journal of Applied Physics 93(9), 5816-5823 (2003).

[6] S. Klose and et. al., Contributions to Plasma Physics 41(5), 467-472 (2001).

[7] M. Ye, S. Takamura, and N. Ohno, Journal of Nuclear Materials 241-243, 1243 - 1247 (1997).

[8] M. Baeva, W. Goedheer, and N. J. L. Cardozo, Plasma Science \& Technology 10(2), 162-169 (2008).

[9] D. Reiter, EIRENE A Monte Carlo linear transport solver, www.eirene.de. 
[10] D. P. Stotler and et. al., J. Nucl. Mater 96(290-293), 967971 (2001).

[11] R. K. Janev and et. al., Elementary Processes in HydrogenHelium Plasmas (Springer-Verlag, 1987).

[12] D. Tskhakaya and S. Kuhn, 29th EPS Conference on Controlled Fusion and Plasma Physics 26B(P-2.094) (2002).

[13] P. L. Bhatnagar, E.P. Gross, and M. Krook, Phys. Rev. 94(May), 511-525 (1954).

[14] M. Capitelli and et. al., Plasma Sources Science and Technology 16(1), S30 (2007). 